\title{
Comparing survival rate and appropriate surgery methods according to tumor location in $\mathbf{T} 2$ gallbladder cancer
}

\author{
Min Kyu SUNG ${ }^{1}$, Dae Wook HWANG*', Bong Jun KWAK², Yejong PARK', Jaewoo KWON', \\ Woohyung LEE', Ki Byung SONG'1, Jae Hoon LEE', Song Cheol KIM' \\ 'Division of Hepatobiliary and Pancreatic Surgery, Department of Surgery, Asan Medical Center, Seoul, Korea \\ ${ }^{2}$ Division of Hepatobiliary, Pancreas, and Abdominal Organ Transplant, Department of Surgery, Incheon St. Mary's Hospital, Incheon, Korea
}

Introduction: The survival outcomes and optimal extent of surgery of T2 gallbladder cancers remain controversial. We aimed to investigate the difference in overall/disease-free survival rates and assess the prognosis of T2 gallbladder cancers.

Methods: We retrospectively reviewed electronic medical records of 147 patients who underwent surgical resection for pathologically confirmed T2 gallbladder cancer between January 2003 and December 2012. Patients were categorized into two groups according to the tumor location (T2a vs. T2b) and three groups according to surgery method (simple cholecystectomy, cholecystectomy with lymph node dissection, and extended cholecystectomy). We compared the overall and disease-free survival rates according to T2 subgroups and surgery methods. Cox proportional hazard analysis was performed to evaluate prognostic factors for the overall survival of T2 gallbladder cancer.

Results: Of all patients, 40 (27.2\%) and 107 (72.8\%) were diagnosed with T2a and T2b gallbladder cancers, respectively. The 5-year overall and disease-free survival rates were $75.0 \%$ vs. $73.8 \%(p=0.653)$ and $72.5 \%$ vs. $70.1 \%(p=0.479)$ in T2a and T2b gallbladder cancers, respectively. There was no difference in the survival rate among T2a gallbladder cancer according to the surgery method. However, in T2b gallbladder cancer, extended cholecystectomy showed a better overall survival than simple cholecystectomy and cholecystectomy with lymph node dissection groups ( $p=0.043$ and $p=0.003$, respectively).

Conclusions: There is no difference in overall and disease-free survival rates according to the location of T2 gallbladder cancers. Extended cholecystectomy increases overall survival rate, especially in T2b gallbladder cancers. 